\title{
Modeling of Multi-View 3D Freehand Radio Frequency Ultrasound
}

\author{
T. Klein ${ }^{1, \star}$, M. Hansson ${ }^{2, \star \star}$, and Nassir Navab ${ }^{1}$ \\ 1 Computer Aided Medical Procedures (CAMP), TU München, Germany \\ 2 Malmö University, School of Technology, Sweden
}

\begin{abstract}
Nowadays ultrasound (US) examinations are typically performed with conventional machines providing two dimensional imagery. However, there exist a multitude of applications where doctors could benefit from three dimensional ultrasound providing better judgment, due to the extended spatial view. 3D freehand US allows acquisition of images by means of a tracking device attached to the ultrasound transducer. Unfortunately, view dependency makes the 3D representation of ultrasound a non-trivial task. To address this we model speckle statistics, in envelope-detected radio frequency ( $\mathrm{RF}$ ) data, using a finite mixture model (FMM), assuming a parametric representation of data, in which the multiple views are treated as components of the FMM. The proposed model is show-cased with registration, using an ultrasound specific distribution based pseudo-distance, and reconstruction tasks, performed on the manifold of Gamma model parameters. Example field of application is neurology using transcranial US, as this domain requires high accuracy and data systematically features low SNR, making intensity based registration difficult. In particular, 3D US can be specifically used to improve differential diagnosis of Parkinson's disease (PD) compared to conventional approaches and is therefore of high relevance for future application.
\end{abstract}

Keywords: Envelope-Detected RF, Ultrasound, Registration, 3D Reconstruction, Finite mixture, Nakagami, View-dependent.

\section{Introduction}

Ultrasonography is often the modality of choice in terms of e.g. intra-operative and screening applications due to its safety, mobility and inexpensiveness compared to other imaging techniques. This together with the steady increase in image quality has made it a widely used diagnostic method in various medical disciplines in recent years. However, the interpretation of US imagery is typically not straightforward and of quite subjective nature and therefore highly dependent on the expertise of its users. This largely stems from the inherent process of US imaging, which is above all view-dependent, as well as subject to noise and

\footnotetext{
* This work was partially sponsored by EU grant FP7-ICT-2009-6-270460.

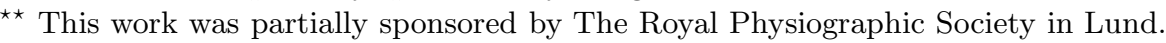


prone to containing various types of artifacts. The characteristic speckle noise, dependent on factors such as spatial arrangement and size of scatterers, forms patterns, which are largely characteristic for various types of tissue. Different statistical models have been proposed to model speckle, including the Rician, generalized K, homodyned K, Rayleigh and Nakagami distribution [1. Specifically in [2], Shankar showed that the envelope detected RF signal follows the Nakagami distribution [3], which serves as a very general model for a multitude of speckle scenarios.

In this paper we extend classical US RF envelope modeling by employing a Nakagami FMM-based approach for 3D US freehand data. This allows us to embed the view-dependent property of US in a statistical formulation. FMMs have already been successfully applied to US data for segmentation of the carotid artery 4. However, our target applications for this model are registration and reconstruction. To our knowledge we are the first to apply a statistical parametric approach in conjunction with similarity measures (in our case the J-divergence distance metric) to registration of 3D US data. Previous works [5] have applied similarity metrics, e.g Kullback-Leibler (KL), Hellinger and Bhattacharyaa, directly without any parametric distributional assumptions on the data.

Performance and use is show-cased on transcranial US (TCUS) brain data, where $3 \mathrm{D}$ freehand image sequences are taken through a narrow bone window at the temporal lobe. The domain of neuro-US is quite relevant for the proposed approach. This is because of the need of high registration accuracy for brain data for applications such as electrode implantation in the brain. Also, TCUS has been recently shown to be appropriate for early diagnosis of PD [6]. This illness is related to degeneration of the substantia nigra (SN). The process is associated with the agglomeration of ferrite deposits, which form hyperechogenic areas in the SN 7] that are visible in US. In this regard, generating 3D data from $2 \mathrm{D}$ images by means of a tracked transducer can help in reducing subjectivity in diagnosis 8. Furthermore, accurate registration of $3 \mathrm{D}$ data is required to perform continuous staging of hyperechogenic SN regions.

\section{Method}

\subsection{Freehand 3D RF Data}

Due to the spatial relationship of the data, the $\mathrm{RF}$ image requires disintegration into individual scanlines such that the reconstruction process, following [9], becomes ray-based - see Fig. 1. Beside the intensity data we also record geometric information such as view point and direction. This additional data is used in a follow-up processing step. The distribution of the envelope of the RF signal, resulting from backscattered tissue echo, has been shown to be modeled, in a simple and versatile way, by the Nakagami distribution [2]. Thus we assume all intensities in the RF envelope image to follow a Nakagami distribution

$$
\mathcal{N}(x \mid \mu, \omega)=\frac{2 \mu^{\mu} x^{2 \mu-1}}{\Gamma(\mu) \omega^{\mu}} \exp \left(-\frac{\mu}{\omega} x^{2}\right) \quad \text { s.t. } \quad \forall x \in \mathbb{R}_{+},
$$

with $\mu, \omega$ denoting the shape and scale parameters, respectively. 


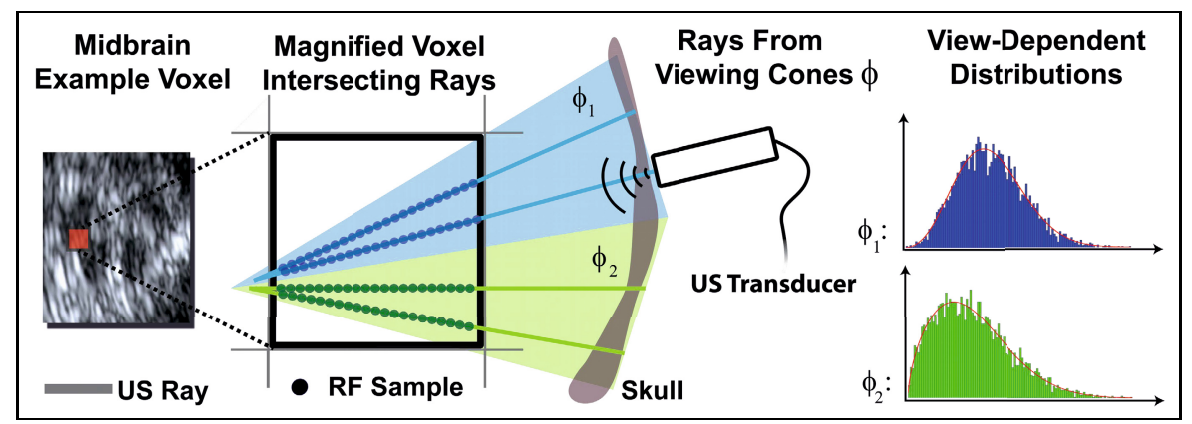

Fig. 1. Distributional change for cones $\phi_{1}$ and $\phi_{2}$ within a single view of midbrain

\subsection{Mixture Model Motivation}

Since ultrasound is highly view-dependent [10, it is desirable to incorporate this property when modeling back-scatter. However, we are not interested in the individual backscatter intensities, but in the distribution within a small finite volumetric element (voxel) w.r.t views of a data point $x$, i.e.

$$
p(x)=\int_{\mathcal{D}(x)} p(x \mid \phi) p(\phi) d \phi
$$

where $\mathcal{D}(x)$ is the set of all possible viewing cones $\phi$ of $x$. The distribution $p(x)$ is approximated by a FMM of $K$ Nakagami densities [4,

$$
p(x) \approx \sum_{k=1}^{K} p\left(\phi_{k}\right) p\left(x \mid \phi_{k}\right)=\sum_{k=1}^{K} w_{k} \mathcal{N}\left(x \mid \mu_{k}, \omega_{k}\right) \quad \text { s.t. } \quad \sum_{k=1}^{K} w_{k}=1
$$

where the distribution $p\left(\phi_{k}\right)$ of the $k^{\text {th }}$ cone is represented by a mixture weight. The $K$ viewing cones are assumed approx. evenly spaced around the object of interest. See Fig. 1 for illustration of viewing cones of the midbrain with beams originating at different skull positions. A popular choice for FMM estimation is the Expectation-Maximization (EM) algorithm [11. However, as the EM algorithm can potentially overfit the data and is also quite flexible in component modeling, we do not instantiate it on the pooled data from all views. Rather, we instantiate individual mixture estimations within geometrical subspaces obtained from each view (each containing viewing cones) that were recorded during the acquisition process. Altogether this yields robust component estimation, s.t.

$$
p(x)=\sum_{i=1}^{N} \sum_{k=1}^{K_{i}} w_{k} p\left(x \mid \phi_{i, k}\right)=\sum_{i=1}^{N} \sum_{k=1}^{K_{i}} w_{k} \mathcal{N}\left(x \mid \mu_{i, k}, \omega_{i, k}\right) .
$$

Specifically, within each view $i \in N$ we determine the number of components $K \leq K_{\max }$, following the approach of Frayley and Raftery [12. Here the Asymptotic Minimum Description Length (AMDL / BIC) principle selects the FMM 
with $d$ free parameters, which minimizes the quantity $-2 \log \mathcal{L}+d \log n$, where $\mathcal{L}$ is the likelihood of data given model parameters and $n$ the number of observations. This estimation process is performed voxelwise in the entire volume, yielding a mixture model representation for each voxel, where the number of components naturally varies from voxel to voxel.

\subsection{Registration}

For registration, we perform a voxel-wise distribution matching employing Jdivergence in conjunction with a data fidelity term. This provides higher robustness compared to a pure intensity-based model, as was also observed in [5]. Considering a fixed volume (A) and moving volume (B), we seek the rigid transformation $\hat{T}$ that yields optimal alignment between the two, s.t. $\hat{T}=$ $\arg \min _{T} D_{\mathrm{PJD}}(A, T(B))$ for the pseudo-distance

$$
D_{\mathrm{PJD}}(C, D)=\underset{i, j}{\arg \min } \sum_{k=1}^{Z} \mathcal{J}\left(f_{C_{k}}^{i}, f_{D_{k}}^{j}\right) \cdot e^{\left(\lambda \cdot\left(\left(1-w_{C_{k}}^{i}\right)+\left(1-w_{D_{k}}^{j}\right)\right)\right)} .
$$

We refer to this pseudo-distance as Pseudo-J-Divergence. For each voxel $k \in Z$ it takes the mixture components pair $f_{C_{k}}^{i}$ and $f_{D_{k}}^{j}$ from the two volumes $C$ and $D$, resp., to be registered with least J-divergence 13 times the exponentially scaled sum of corresp. mixture weights $w_{C_{k}}^{i}, w_{D_{k}}^{j} \geq \tau$, where $\tau$ is the min. weight. Lastly, $\lambda$ is a parameter. The pseudo-distance $D_{\mathrm{PJD}}$ does not satisfy the triangle inequality, but inherits symmetricity and uniqueness from the J-divergence $\mathcal{J}(f, g)$. The exponential weight in (5) punishes distances formed from components with low mixture weights, since these components are assumed to be less descriptive of the underlying distribution. The J-divergence between two Gamma distributions $f, g \in \mathcal{G} \mathcal{A}$, is the sum of two non-symmetric KL distances with switched arguments, s.t.

$$
\begin{aligned}
\mathcal{J}(f, g) & =\int \log \frac{f(x)}{g(x)}(f(x)-g(x)) d x=\left(\mu_{a}-1\right) \Psi\left(\mu_{a}\right)-\log \omega_{a}-\mu_{a} \\
& -\log \frac{\Gamma\left(\mu_{a}\right)}{\Gamma\left(\mu_{b}\right)}+\mu_{b} \log \omega_{b}-\left(\mu_{b}-1\right)\left(\Psi\left(\mu_{a}\right)+\log \omega_{a}\right)+\frac{\omega_{a} \mu_{a}}{\omega_{b}}
\end{aligned}
$$

Although the data follows Nakagami distribution, the Gamma distribution may be used instead, as they are related by a simple transformation, given by $Y \sim$ $\mathcal{G} \mathcal{A}\left(x \mid \mu_{\text {gam }}, \omega_{\text {gam }}\right), X \sim \mathcal{N}\left(x \mid \mu_{n a k}, \omega_{n a k}\right) \Rightarrow \sqrt{X}=Y\left(\mu_{n a k}, \omega_{n a k} / \mu_{n a k}\right)$.

\subsection{Reconstruction}

Given the voxel FMM representation we can perform a novel type of reconstruction. Therefore a reference component is chosen from the mixture for each

\footnotetext{
${ }^{1}$ Jeffreys $(\mathrm{J})$ divergence is also known as symmetric KL distance.
} 
voxel, on basis of maximum mean intensity, although other approaches are conceivable. However, this criterion guarantees that no high intensity backscatter is missed during reconstruction. Note that artifacts, such as shadows, might require specific treatment, which is beyond the scope of this work. By optimizing the reference component parameters, i.e. minimizing the sum of geodesic distances to neighbours component parameters, on the manifold $\mathcal{G}$ of Gamma model parameters, smoothness of the reconstructed volume is achieved. Thus, given a point $\theta_{a}=\left(\mu_{a}, \omega_{a}\right) \in \mathcal{G}$, the geodesic distance $D_{\text {geo }}$ to a locally neighbouring point $\theta_{b}=\left(\mu_{b}, \omega_{b}\right) \in \mathcal{G}$ is bounded s.t.

$$
D_{g e o}\left(\theta_{a}, \theta_{b}\right) \leq\left|\frac{d^{2} \log \Gamma}{d \mu^{2}}\left(\mu_{b}\right)-\frac{d^{2} \log \Gamma}{d \mu^{2}}\left(\mu_{a}\right)\right|+\left|\mu_{a} \cdot \log \frac{\mu_{a} \omega_{a}}{\mu_{b} \omega_{b}}\right|,
$$

cf. Ch. 7, 14 and 15. Applying (7), the reference distribution $\theta_{\text {ref }}$ is optimized by minimizing the sum of geodesic distances to all of its neighbors s.t.

$$
\hat{\theta}_{\text {ref }}=\underset{\theta_{\text {ref }}}{\operatorname{argmin}} \sum_{k \in N_{\text {ref }}} D_{g e o}\left(\theta_{\text {ref }}, \theta_{k}\right)
$$

keeping the neighbours $\theta_{k} \in N_{\text {ref }}$ fixed, where $N_{\text {ref }}$ defines the neighborhood of a reference voxel. This yields a spatially consistent image without over-smoothing or loss of detail in terms of highlights. Typically a few optimization steps are sufficient and allow for a fast reconstruction.

For data reconstruction within a voxel we apply a Gaussian-weighted (GW) [9] reconstruction scheme in order to increase homogeneity,

$$
y_{j}=\frac{1}{Z} \sum_{i=1}^{N} x_{i} e^{-d_{i}^{2} / \sigma^{2}} \quad \text { s.t. } \quad Z=\sum_{i=1}^{N} e^{-d_{i}^{2} / \sigma^{2}},
$$

yielding the reconstructed intensity $y_{j}$ at voxel position $\mathrm{j}$. Here the intensities $x_{i}$ are sampled from the reference distribution, where $d_{i}$ are the distances from the voxel centroid that can be obtained by regression from the measured data.

\section{Results}

Registration. The 3D US RF freehand data used is obtained using the optical tracking system NDI Spectra in conjunction with an Ultrasonix MDP US machine. For testing the registration performance multiple transcranial 3D volumes for several patients were acquired. An acquisition consisted of bilateral scans; in doing so various sweeps from numerous possible views were obtained. Furthermore, each patient was recorded with a reference target rigidly attached to the head, which allows establishment of groundtruth position between numerous volumes. The three-dimensional RF datasets were acquired using a phased-array probe with a frequency of $3.3 \mathrm{MHz}$ and depth $14 \mathrm{~cm}$. RF data is sampled at 40 $\mathrm{MHz}$. Each 3D RF data set is built from approximately 4000 2D RF images (2000 images from each side of the skull), each having a resolution of 3648 x 96 pixels. 


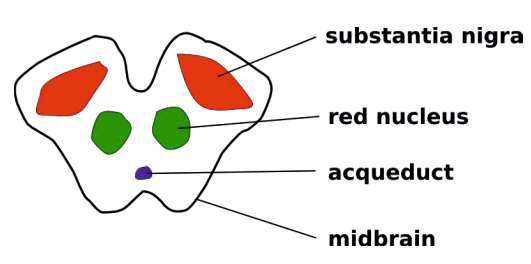

Fig. 2. Schematic illustration of midbrain and structures within
Table 1. Median errors (mm) and std. dev. of registration study

\begin{tabular}{|l|c|c|c|}
\hline Dataset & SSD & NCC & PJD \\
\hline$\# 1$ & $3.2 \pm 1.3$ & $3.0 \pm 1.3$ & $\mathbf{2 . 7} \pm \mathbf{1 . 1}$ \\
$\# 2$ & $2.7 \pm 1.3$ & $2.7 \pm 1.2$ & $\mathbf{2 . 4} \pm \mathbf{1 . 2}$ \\
$\# 3$ & $3.2 \pm 1.2$ & $3.3 \pm 1.1$ & $\mathbf{2 . 9} \pm \mathbf{1 . 1}$ \\
$\# 4$ & $3.7 \pm 1.1$ & $3.4 \pm 1.1$ & $\mathbf{3 . 0} \pm \mathbf{1 . 1}$ \\
$\# 5$ & $3.4 \pm 1.1$ & $3.2 \pm 1.1$ & $\mathbf{2 . 9} \pm \mathbf{1 . 1}$ \\
$\# 6$ & $3.3 \pm 1.2$ & $3.4 \pm 1.2$ & $\mathbf{2 . 9} \pm \mathbf{1 . 1}$ \\
\hline
\end{tabular}

Acquisition time is 2-4 min (1800 images/min unilateral scan). Volumes were reconstructed with isotropic voxel size of $0.65 \mathrm{~mm}$ (mean 1400 samples/voxel). For the mixture modeling we assumed $N=2$ views (bilateral) as well as $K_{\max }=2$. This yielded a maximum of four mixture components per voxel and sufficiently modeled the data while avoiding overfits.

For evaluating the quality of the proposed approach, we performed registration by block matching, which is commonly used for US 1617. For each patient two multi-view volumes are constructed for distinct US data. These two distinct volumes are then rigidly registered by taking 27 equally distributed blocks (each of size $6 \times 6 \times 6$ voxels) for matching, within each multi-view volume. For each of 10 runs we randomly displace, with initial deviation of $\pm 6 \mathrm{~cm}$, the moving subvolume/block from the ground truth position (obtained via head target), in each spatial direction from its ground truth position. This is followed by registration by block-matching at each position using state of the art similarity metrics for US, aligning the moving and the fixed volume. In the case of global registration, the result from each block is accumulated. Parameters for our distance metric were set to $\lambda=2$ and $\tau=0.3$. In spite of rigidity, registration of transcranial brain US brain data is quite challenging due to the relative low SNR as a result of variable transmission through skull bone and the low transducer frequency required. Nevertheless, the proposed pseudo-J-Divergence (PJD) yields up to $15 \%$ better registration results compared to Normalized Cross-Correlation (NCC) and Sum of Squared Differences (SSD) [1617, as can be seen in Tab. 1. Intensity volumes were created using GW [9]. The difference in median between methods is statistically significant. The Hodges-Lehmann 95\% confidence interval for the difference in median error between PJD and NCC is $(-0.25,-0.08)$, and $(-0.3,-0.12)$ for PJD and SSD. The corresp. Mann-Whitney U-test yields p-values $0.14 \cdot 10^{-3}$ and $0.2 \cdot 10^{-6}$, resp. Obtained results are quite close to technical possible limits due to the errors that are propagated through the processing chain. In this respect, US calibration affects the accuracy significantly, depending largely on the approach used. In our setup, calibration was performed using a single wall phantom, yielding an error between 1-2 mm [1819.

Reconstruction. As reconstruction methods are difficult to compare due to the highly subjective nature of US image analysis, we will elaborate on the obvious differences between a state of the art method (GW - increased smoothness 

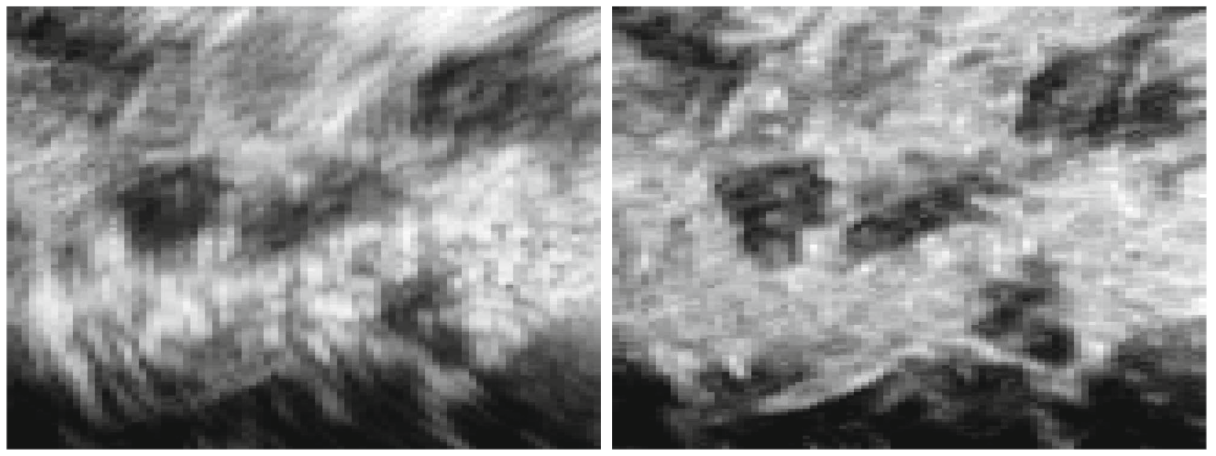

Fig. 3. TCUS reconstruction. Butterfly-shaped region in the image center corresponds to the midbrain. Left: Gaussian-weighted Right: Geodesic.

favourable for segmentation and registration [9]) and our approach as well as discuss the potential implications. Reconstructions following the geodesic approach yield more coherent and homogeneous images - see Fig. 3. Note that for Gaussian reconstruction we chose $\sigma=1$ in all cases. Checker-board and radial artifacts disappear, which are visible in the GW approach due to the multi-view mixture nature of the signal. Additionally, the mid-brain area exhibits sharper edges using the proposed approach. Regions that are clinically relevant for classification such as the SN, where the level of hyperechogenicity is a risk-assessment criterion for PD [6], are more pronounced and less blurred (see Fig. 2 for a schematic illustration of the midbrain and structures within). The clinical benefit for applications such as classification, however, remains to be evaluated.

\section{Conclusion}

We have presented a FMM representation of 3D RF data exploiting its viewdependent statistical properties. Making use of view-dependency has potential to facilitate several applications; specifically, results from a block-matching based rigid registration study suggest improvements in terms of accuracy compared to conventional similarity metrics. Moreover, image reconstruction promises to be an interesting domain of application. Further applications will be studied e.g. multi-view bone imaging where, as a result of varying reflectance properties of bone, muscle, and ligaments, different backscatter scenarios are encountered.

\section{References}

1. Destrempes, F., Cloutier, G.: A Critical Review and Uniformized Representation of Statistical Distributions Modeling the Ultrasound Echo Envelope. Ultrasound Med. Biol. 36(7), 1037-1051 (2010)

2. Shankar, P.M.: A General Statistical Model for Ultrasonic Scattering from Tissues. IEEE Trans. Ultrason. Ferroelectr. Freq. Control 47(3), 339-343 (2000) 
3. Nakagami, N.: The m-distribution, a General Formula for Intensity Distribution of Rapid Fadings. In: Hoffman, W.G. (ed.) Statistical Methods in Radio Wave Propagation, pp. 3-36. Pergamon, Oxford (1960)

4. Destrempes, F., Meunier, J., Giroux, M.F., Soulez, G., Cloutier, G.: Segmentation in Ultrasonic B-Mode Images of Healthy Carotid Arteries Using Mixtures of Nakagami Distributions and Stochastic Optimization. IEEE Trans. Med. Imag. 28(2), 215-229 (2009)

5. Ijaz, U.Z., Prager, R.W., Gee, A.H., Treece, G.M.: A Study of Similarity Measures for In Vivo 3D Ultrasound Volume Registration Acoustical Imaging. Acoustical Imaging, vol. 30, pp. 315-323. Springer, Netherlands (2011)

6. Walter, U., Dressler, D., Probst, T., Wolters, A., Abu-Mugheisib, M., Wittstock, M., Benecke, R.: Transcranial Brain Sonography Findings in Discriminating Between Parkinsonism and Idiopathic Parkinson Disease. Arch. Neurol. 64(11), 16351640 (2007)

7. Becker, G., Seufert, J., Bogdahn, U., Reichmann, H., Reiners, K.: Degeneration of Substantia Nigra in Chronic Parkinson's Disease Visualized by Transcranial Colorcoded Real-time Sonography. Neurology 45(1), 182-184 (1995)

8. Plate, A., Ahmadi, S.-A., Klein, T., Navab, N., Weisse, J., Mehrkens, J.H., Boetzel, K.: Towards a More Objective Visualization of the Midbrain and its Surroundings Using 3D Transcranial Ultrasound. In: Deutschen Gesellschaft für Klinische Neurophysiologie und Funktionelle Bildgebung (DGKN), Halle, Germany (2010)

9. Wein, W., Pache, F., Röper, B., Navab, N.: Backward-Warping Ultrasound Reconstruction for Improving Diagnostic Value and Registration. In: Larsen, R., Nielsen, M., Sporring, J. (eds.) MICCAI 2006, Part II. LNCS, vol. 4191, pp. 750-757. Springer, Heidelberg (2006)

10. Hedrick, W.R., Hykes, D.L., Starchman, D.E.: Ultrasound Physics and Instrumentation. Mosby (2004)

11. Dempster, A.P., Laird, N.M., Rubin, D.B.: Maximum Likelihood for Incomplete Data via the EM Algorithm. J. Roy. Statist. Soc. Ser. B 39, 1-38 (1977)

12. Fraley, C., Raftery, A.E.: Model-based Clustering, Discriminant Analysis, and Density Estimation. J. Amer. Statist. Assoc. 97(458), 611-631 (2002)

13. Jeffreys, H.: An Invariant Form for the Prior Probability in Estimation Problems. Proc. R. Soc. A. Series A, Mathematical and Physical Sciences 186, 453-461 (1946)

14. Arwini, K., Dodson, C., Doig, A., Sampson, W., Scharcanski, J., Felipussi, S.: Information Geometry: Near Randomness and Near Independence. Lect Notes Math. Springer, Heidelberg (2008)

15. Dodson, C., Matsuzoe., H.: An Affine Embedding of the Gamma Manifold. InterStat, 1-6 (2002)

16. Poon, T.C., Rohling, R.N.: Three-dimensional Extended Field-of-view Ultrasound. Ultrasound Med. Biol. 32(3), 357-369 (2006)

17. Krucker, J.F., LeCarpentier, G.L., Fowlkes, J.B., Carson, P.L.: Rapid Elastic Image Registration for 3-d Ultrasound. IEEE Trans. Med. Imag. 21(11), 1384-1394 (2002)

18. Hsu, P.W., Prager, R.W., Gee, A.H., Treece, G.M.: Freehand 3D Ultrasound Calibration: A Review (2007)

19. Mercier, L., Lango, T., Lindseth, F., Collins, D.: A Review of Calibration Techniques for Freehand 3-d Ultrasound Systems. Ultrasound Med. Biol. 31(4) (2005) 